\title{
Tunnels sous-marins pour le refroidissement des centrales nucléaires
}

\author{
PAR
}

\author{
P. Duffaut \\ Ingénieur Civil des Mines \\ Électricité de France, Equipement, \\ Division Techniques d'Exécution
}

Lorsqu'il faut raccorder une centrale construite sur le bord de mer à une prise d'eau située au large, de nombreuses solutions sont en concurrence. Le canal limité par des digues et le pont-canal, qui est une conduite portée au-dessus de la mer, sont traités par ailleurs (Mainier et Lepetit, Lacroix). Les conduites flottantes et les conduites immergées, posées sur le fond ou ensouillées, ont été considérées comme difficiles à exécuter et à entretenir en raison des conditions de vagues et de courants propres au voisinage immédiat des côtes. Au contraire, la solution souterraine a, dès l'abord, retenu l'attention: le projet d'un tunnel est de loin le plus simple, l'exploitation d'un tunnel ne requiert qu'un entretien intérieur, commun à toutes les solutions, et si lexécution peut poser des problèmes. les vagues et courants n'y ont pas de part. Le projet et l'exécution seront examinés successivement ci-dessous, puis l'application au cas particulier de la centrale de $\mathrm{Pa}$ luel. Les problèmes posés par les prises d'eau et, plus généralement, la mise en communication des tunnels avec la mer ne sont pas traités ici.

\section{Projet d'un tunnel sous la mer}

Par hypothèse. la solution souterraine est limitée à un terrain rocheux où le projet ne diffère pas de celui d'un tunnel à vocation hydroélectrique.

Toutefois, la pression de l'eau souterraine est souvent négligée dans le calcul des tunnels hydroélectriques. Si le tunnel est sous une nappe d'eau, c'est le niveau de cette nappe qui va commander la piézométrie.

Il convient ici de tenir compte de cette pression en sus des charges dues au terrain supposé sec. Cette opinion doit être toutefois doublement nuancée :

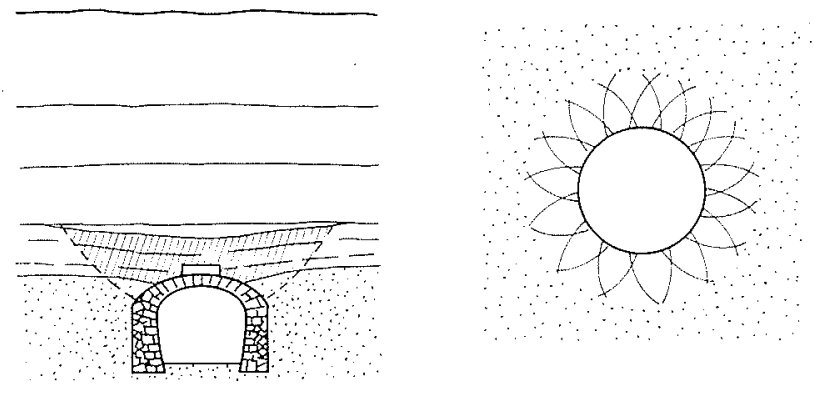

1 / Charge réellement exercée par le terrain, polds de la zone désorganisé par les travaux au-dessus d'un tunnel pou profond (exemple tunnel SNCF de Vierzy); ou bien confinement d'un anneau plastifís autour d'un tunnes très profond.

a) la pleine pression hydrostatique de l'eau ne s'exerce que sur le revêtement parfaitement étanche d'un tunnel vide.

Le poids du terrain à prendre en compte dans ce cas est alors au plus le poids déjaugé puisque ce terrain baigne entièrement dans l'eau. Ceci revient à diminuer de 1 sa densité ( $\left.{ }^{1}\right)$, ce qui représente un allègement considérable, compris entre $1 / 3$ pour les roches les plus lourdes, basaltes compacts par exemple, et $1 / 2$ ou même jusqu'à $2 / 3$ pour les roches poreuses et les sols.

En réalité, à l'exception de tunnels très superficiels dans des terrains pratiquement pulvérulents, le revêtement ne supporte jamais le poids du terrain ni généralement l'effet du tenseur contrainte initiale dans le milieu solide (fig. 1) : ou bien il supporte le poids d'une petite épaisseur de terrain désorganisé par les travaux, ou bien il contient la dilatance d'une mince zone brisée ou plastifiée, en lui pro-

(1) Densité apparente à sec. 
curant le léger confinement qui assure sa stabilité. La pression entre terrain et revêtement n'étant jamais qu'une petite fraction de la contrainte initiale dans le terrain, l'effet supplémentaire de l'eau est essentiel.

b) Cette pression exercée par l'eau, à l'extérieur du revêtement, doit d'abord être diminuée de la pression de l'eau à l'intérieur. En exploitation normale des tunnels de prise d'eau en mer, la différence de pression sera minime entre l'intérieur et l'extérieur, et pour cette raison l'étanchéité du revêtement n'a pas besoin d'être rigoureuse.

Supposant le revêtement perméable, l'eau s'écoule au travers mais aussi dans tout le massif encaissant; la chute de pression de l'eau ne se concentre donc pas sur le seul extrados du revêtement mais se répartit dans son épaisseur et dans tout le massif; le revêtement n'en supporte directement qu'une fraction qui dépend du champ de perméabilité dans l'ensemble terrain + revêtement (fig. 2). Le schéma est le même que pour les barrages en terre : la pression hydrostatique s'exercerait entière sur un masque ou un noyau parfaitement étanche, mais elle se diffuse dans toute la masse d'un barrage homogène, en se concentrant toutefois davantage à l'approche du filtre où les équipotentielles sont plus serrées; elle se répartit entre la masse plus perméable et le noyau moins perméable dans le cas général.

Si la pression directe de l'eau sur le revêtement est ainsi très fortement diminuée, il faut prendre en compte un effet indirect puisque la chute de pression de l'eau est transférée au massif rocheux sous forme de pression d'écoulement le long des lignes de courant; la pression du terrain sur le revêtement peut donc en être augmentée (Rat 1973). Ce

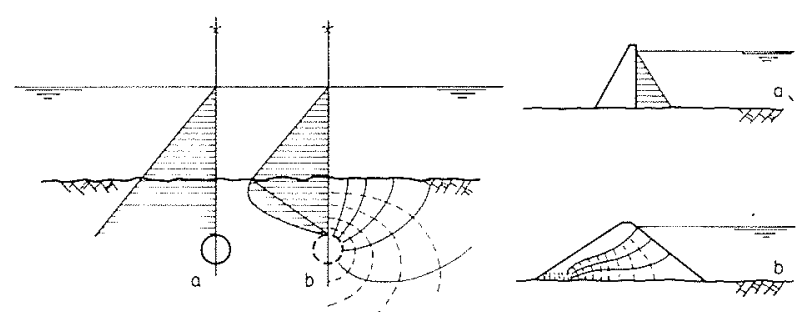

2 / Charges exercées par l'eau sur un tunnel el sur un barrage: a) sans écoulement, la pleine poussée hydrostatique est concentrée sur l'organe d'étanchélté,

b) avec écoulement, la perte de charge est répartie le long des llgnes de courant en fonction de l'espacement relatif des équipotentielles.

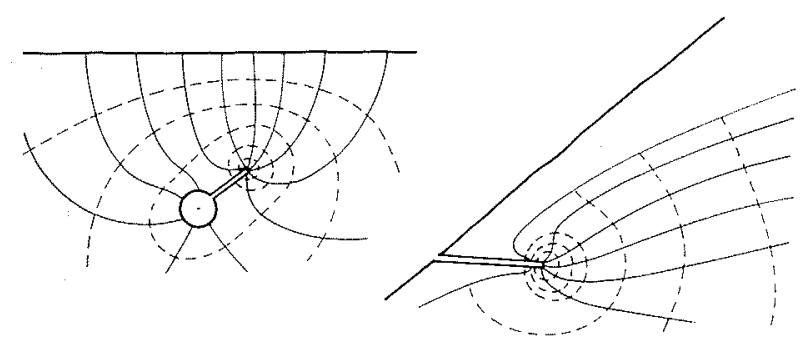

3 / Un drainage prétérentlel par flssure ou par torage ecarte les gradlents élevés de la surface libre, qu'il s'aglsse d'un tunnel ou du pled d'un versant. phénomène, et plus précisément le régime transitoire qui précède l'établissement d'un régime permanent après la construction d'un revêtement, pourrait être la principale explication de l'augmentation progressive des efforts supportés par les revêtements de tunnels (alors qu'elle est parfois mise sur le compte d'un comportement viscoélastique du terrain).

Bien entendu, et comme dans le cas du barrage, la pleine pression hydrostatique est une borne supérieure pour la pression totale produite par l'eau sur le revêtement, directement et indirectement. (En particulier, dans le cas du barrage poids en béton, la prise en compte d'un écoulement à travers le béton conduirait à une résultante des forces dues à l'eau plus favorable à la stabilité).

Mais le calcul théorique de l'écoulement de l'eau autour du tunnel est très pessimiste parce qu'il suppose une symétrie de révolution tout à fait improbable et en outre instable. Le moindre écart à cette symétrie privilégiera un cheminement le long duquel la zone de gradient hydraulique élevé se trouvera repoussée au large du tunnel (fig. 3).

L'effet mécanique de l'écoulement sur le terrain sera le plus souvent complètement annulé. On pourrait d'ailleurs obtenir théoriquement le même effet à volonté par des forages de drainage, jouant le même rôle stabilisateur que le drainage du pied d'un versant ou le filtre d'un barrage. Cette observation n'est valable que pour la période des travaux car, en exploitation, la maintenance de ces drains ne pourrait être assurée.

Le même raisonnement sur l'improbabilité d'une symétrie de révolution s'applique, d'ailleurs, pour diminuer les contraintes dans le rocher au périmètre d'une cavité souterraine. La moindre hétérogénéité ou discontinuité permet une adaptation du périmètre entier.

\section{Tableau 1}

\section{DÉBITS RENCONTRÉS EN SOUTERRAIN}

1. Tumnels ferroviaires anciens :

$\left(\mathrm{m}^{3} / \mathrm{s}\right)$

Saint-Gothard . . . . . . . . . . . 0,2

Simplon .................. 1,2

Col de Tende ................ 1,1

Mont d'Or............. 3 à 10

2. Tumnels hydroélectriques d'E.D.F. :

Ponturin .................. 1,8

Versoyen .................... 6

La Coche $(11$ avril 1975) ........ 2 descendu en 1 mois à ......... 0,15

en 4 mois à $\ldots \ldots \ldots \ldots, 0,08$

3. Tumnel hydroélectrique au Liban :

Jezzine amont $\ldots \ldots \ldots \ldots \ldots \ldots \ldots$ 0,7 descendu en 6 mois à .......... 0 remonté brutalement à ........ 6 retombé en 12 heures à......... 3 en 15 jours à $\ldots \ldots \ldots \ldots, 0,1$ 

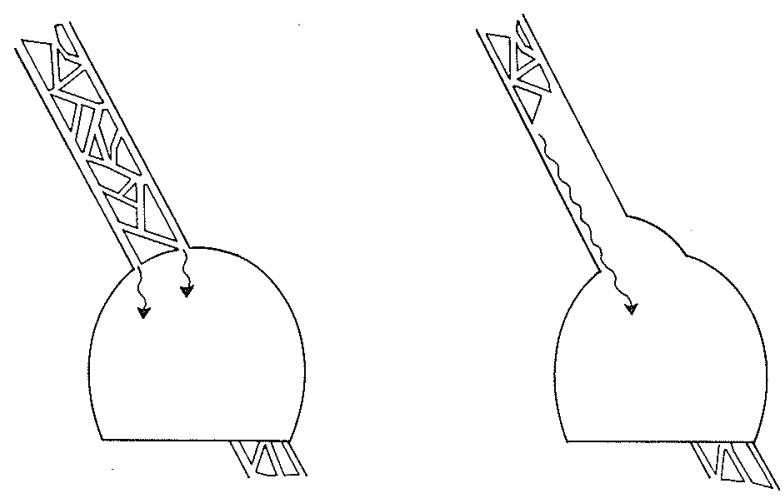

4 / Evolution d'une zone falliée aqulfère en cheminée dès que le premler bloc est entrainé.

Exemple chiffré: pour un tunnel de $5 \mathrm{~m}$ de diamètre à $40 \mathrm{~m}$ sous la mer, si la compression admissible dans le béton est $10 \mathrm{MPa}$, l'épaisseur théorique utile d'un tube étanche est $0.1 \mathrm{~m}$ en ne tenant compte que de l'eau (formule du tube mince).

En pratique, on utilise toujours des épaisseurs supérieures et pourtant, en service normal, la différence ne dépassera guère $4 \mathrm{~m}$ d'eau : la compression dans le béton sera seulement alors $1 \mathrm{MPa}$.

\section{Exécution d'un tunnel sous la mer}

Creuser sous la mer n'est pas nouveau, puisque les mineurs y ont depuis longtemps suivi les filons ou les couches qu'ils avaient commencé à exploiter sous les terres émergées, dans les mines d'étain des Cornouailles dès l'époque romaine, dans les mines de fer de Diélette, au large du Cotentin, fermées depuis quelques années seulement. et prochainement sous la mer du Nord où se prolonge l'un des principaux bassins houillers d'Angleterre. En réalité, le sous-sol marin se présente au mineur exactement comme le sous-sol terrestre, dès lors que la surface de la nappe phréatique y a été franchie : c'est l'eau souterruine qui est dans les deux cas le principal obstacle à l'exécution des travaux souterrains; sous la mer, on peut dire seulement qu'il serait moins pardonnable de l'oublier.

La différence principale entre travaux sous terre et sous mer n'est pas dans la pression de l'eau, mais dans ses conséquences et notamment l'évolution possible du débit. La pression hydrostatique initiale peut atteindre $50 \mathrm{~m}$ d'eau à $50 \mathrm{~m}$ sous la mer, mais les tunnels exécutés sous les montagnes ont rencontré des pressions beaucoup plus fortes, bien souvent les $2 / 3$ ou les $3 / 4$ de la hauteur de couverture, par exemple $150 \mathrm{~m}$ d'eau à la fenêtre 13 de Malgovert: $500 \mathrm{~m}$ à Sainte-Anne, galerie en charge du Mont-Cenis, et même $750 \mathrm{~m}$ à Awali (Liban) sous $700 \mathrm{~m}$ de couverture (ce dépassement pouvant être justifié par la coupe du terrain qui s'élevait davantage à peu de distance du tunnel) (Halwani et al, 1965).

Quant au débit instantané rencontré sous les montagnes, il est parfois considérable, mais il ne se conserve généralement pas (tableau 1). Il est arrivé qu'un tunnel en terrain karstique crève un véritable petit lac souterrain; une fois celui-ci vidé, le débit se stabilise au niveau de la seule alimentation régulière de ce lac. Cette même image est valable dans le cas général: les forts débits initiaux correspondent à une conductivité des fractures supérieure à la moyenne dans une zone limitée et, après un certain délai, c'est la conductivité moyenne du massif qui règle le débit du régime permanent à un niveau bien inférieur. En outre, la nappe initiale est déprimée par le drainage et, même en milieu homogène, le débit initial diminue en conséquence.

Si toutefois la zone à forte conductivité s'étend jusqu'à une importante nappe d'eau superficielle, lac, mer ou cours d'eau, aucune diminution du débit initial ne peut être attendue et, bien au contraire, l'entraînement du solide le long des cheminements les plus courts, où le gradient est le plus élevé, peut contribuer à augmenter les sections de passage, donc les débits. Il est clair que c'est le vrai problème du tunnel sous-marin: l'augmentation indéfinie du débit, autorisée par le volume disponible indéfini, conduit à noyer le chantier quels que soient les moyens d'exhaure mis en cuvre.

Ainsi, dans un sol meuble, c'est exactement la même condition dé renard qui s'applique à ce problème et à celui des digues et barrages. Encore la pesanteur s'exerce-t-elle au-dessus du tunnel dans la direction la plus défavorable. Même dans un terrain rocheux fracturé, les blocs assez gros peuvent être délogés par le courant, en commençant justement par le plus proche de la paroi. Ce développement de l'érosion régressive le long des venues d'eau est classique bien que son explication ne soit pas toujours clairement acceptée. Il conduit souvent à des "cheminées" de plusieurs mètres de hauteur, qui s'arrêtent lorsqu'un bloc un peu plus gros reste coincé (fig. 4).

Ce phénomène de destruction du terrain n'est pas directement lié au débit mais seulement à la pression ou plus exactement, au gradient de pression au voisinage de la paroi $\left({ }^{2}\right)$. En effet, on retrouve ici le même phénomène d'instabilité du terrain que dans la vidange rapide des barrages et dans le creusement rapide d'une excavation au pied d'un versant où la nappe est proche : au début du régime transitoire déclenché par le creusement ou la vidange, et avant l'établissement d'un régime d'écoulement permanent, c'est la pression initiale de l'eau qui règne au

(2) II peut se produire même en l'absence d'écoulement visible dans des terrains argileux suffisamment peu perméables pour que le débit écoulé s'évapore en totalité. 
voisinage immédiat de la paroi du tunnel, du versant ou du barrage, et le gradient est donc presque infini, il est d'autant plus grand que le tunnel avance vite, et pour une vitesse d'avancement donnée, il agit d'autant plus longtemps que la conductivité est faible.

C'est pour neutraliser ce dangereux mécanisme que les précautions suivantes doivent être strictement appliquées:

- proscrire absolument les tunnels dans les sols meubles. ou bien rendre ces sols sûrs par congélation;

- reconnaître, par sondages à l'avancement systématiques, les fractures à remplissage meuble, afin de les obturer par injections avant de les recouper à pleine section;

- surveiller toute venue d'eau et mettre en cuvre leur étanchement s'il y a une augmentation du débit;

- éviter toute progression de cheminée en terrain rocheux fracturé, tant par soutènement direct que par capture de l'eau au-delà de la partie vulnérable que constitue la paroi du tunnel;

- enfin, limiter le gradient hydraulique moyen dans le terrain au-dessous de 1, ou exceptionnellement 2, en terrain très favorable, le gradient étant exprimé sans dimension comme quotient de la hauteur d'eau par la longueur de cheminement.

Une méthode générale pour réduire le gradient est le travail sous air comprimé, classique jusqu'à $10 \mathrm{~m}$ d'eau ou un peu plus, mais inacceptable à partir de $20 \mathrm{~m}$ en raison des délais de compression.

Une précaution supplémentaire est le cloisonnement du chantier par des portes étanches pour limiter la zone abandonnée en cas d'échec du traitement d'une venue d'eau.

\section{Application à Paluel}

Le site choisi est proche de Saint-Valéry-en Caux, entre Étretat et Fécamp (Seine-Maritime). Les bâtiments seront encastrés dans la falaise de craie, une valleuse naturelle limitant le volume de terrassements nécessaire.

Le projet comporte quatre tunnels parallèles, un pour chaque tranche de $1300 \mathrm{MW}\left({ }^{3}\right)$ :

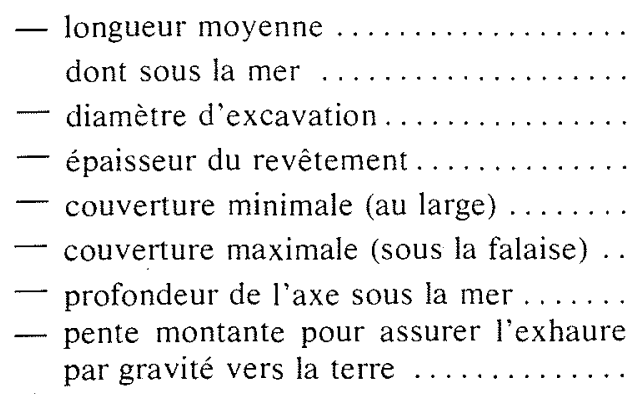

$\begin{array}{cc}1000 & \mathrm{~m} \\ 750 & \mathrm{~m} \\ 5 & \mathrm{~m} \\ 0,4 & \mathrm{~m} \\ 20 & \mathrm{~m} \\ 100 & \mathrm{~m} \\ 38 & \mathrm{~m} \\ 0,1 & \%\end{array}$

La couverture rocheuse minimale est donc de 4 diamètres, et le gradient hydraulique moyen atteint 0,75 , en supposant le régime permanent établi en terrain homogène.

(3) Ce projet est encore susceptible d'importantes modifications.
Ce qui a conduit le projeteur à ces dispositions, assez audacieuses pour une première réalisation, c'est que le terrain parait favorable. Ce terrain est une craic à silex, en bancs presque horizontaux, datée du sénonien inférieur.

Depuis 1967, Électricité de France a eu l'occasion de faire plusieurs études sur diverses craies (Comes, 1973): d'abord, pour le projet (abandonné depuis) d'un aménagement de transfert d'énergie par pompage à Venteuil à côté d'Épernay. (Les études exécutées à cette occasion ont été exposées à la S.H.F. par Dessenne et Duffaut en 1970.) Parmi les autres maitres d'ouvrage qui se sont intéressés aux craies. on peut citer les Ponts-et-Chaussées, qui ont organisé un symposium à Rouen en 1971: Situmer, qui a procédé à d'importants essais en place à Sangatte (Plouviez et al, 1974); Géostock, qui creuse actuellement un important stockage souterrain près de Gargenville (près de Rouen, un stockage de propane liquéfié est en exploitation depuis 1969.)

Par rapport à l'ensemble des craies, la craie de Paluel a des caractéristiques physiques qui la placent plutôt au bas de l'échelle des résistances:

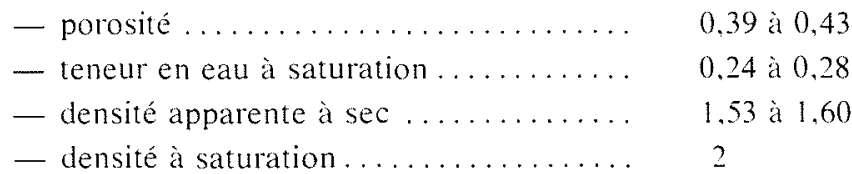

Les résistances obtenues sont assez sensiblement anisotropes, plus fortes dans la direction verticale. Les résistances des échantillons saturés obtenus à partir des forages de reconnaissance sont :

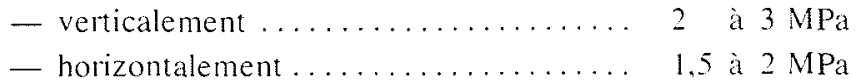

Un critère de qualité de la craie a été proposé par Masson (1973). c'est le rapport entre la résistance de la craie sèche et celle de la craie saturée, en compression simple. Ce rapport varie entre 1 et 5 , il est compris ici entre 3 et 4 suivant les échantillons.

Ces valeurs font de la craie une roche éminemment facile à creuser, et c'est bien pourquoi la première machine foreuse digne de ce nom a pu creuser. en 1882, les tunnels préparatoires au tunnel sous la Manche. Toutefois, il s'agissait de craie sans silex. Dans les falaises de Paluel. les silex forment, tous les 60 ou $80 \mathrm{~cm}$, des lits abondants et parfois continus dont l'épaisseur peut dépasser $20 \mathrm{~cm}$. mais, au niveau du tunnel, ils sont beaucoup plus dispersés. On envisage donc l'emploi de machines ponctuelles en espérant que les pics du tambour seront capables de briser les silex.

Mais réciproquement cette faible résistance peut posel un problème de stabilité des parois.

Dans l'hypothèse très simplifiée d'un problème élastique de révolution, la contrainte à la paroi est égale à deux fois la pression initiale. La résistance à la compression minimale de $15 \mathrm{MPa}$ correspond donc à une pression hydrostatique de $0,75 \mathrm{MPa}$, c'est-à-dire à $75 \mathrm{~m}$ d'eau ou à $37,5 \mathrm{~m}$ de terrain seulement. La résistance moyenne de $25 \mathrm{MPa}$ donne une marge jusqu'à $62,5 \mathrm{~m}$.

Au-delà de ces couvertures, on pourrait constater les mêmes phénomènes d'écaillage, qui ont considérablement compliqué le creusement du tunnel du Mont-Blanc, et qui ont été retrouvés dans la craie sénonienne de Champagne 
lorsque le tunnel de reconnaissance de Venteuil a dépassé $100 \mathrm{~m}$ de couverture (Duffaut et Piraud, 1975)

Le tracé sous la mer est à l'abri de ces phénomènes. mais le franchissement du bord de la falaise correspond a une couverture de 60 à $100 \mathrm{~m}$ (suivant les tunnels).

Un tunnel de reconnaissance est actuellement en cours. Il a permis de prélever des échantillons dans de meilleures conditions qu'en forages et les résistances sont plus élevées. Il est prévu d'y effectuer des mesures de contrainte et de déformation et d'y essayer diverses méthodes de soutènement, notamment le béton projeté et les boulons d'ancrage.

\section{Références}

CoNes (G.), - La craie dans les sites douvrages de production d'électricité (1973). Bull. Liaison Labo P.-et-Ch., n" spécial $\checkmark$ (entièrement consacré à la craie).
Dessevine (J.-L.) et Duffaut (P.). - Les propriétés rhéologiques de la craie et leur influence sur le percement des galeries (1970). La Houlle Blanche, mai 1970.

Duffaut (P.) et PIRAud (J.). - Soutènement des tunnels profonds, autrefois et aujourd'hui (1974). Cahier du Comite Français de Mécanique des Roches, Industrie minérale, n" spécial, 15 décembre 1975

HALWANI, JANOD et LAJEAT. - Le percement du tunnel d'Awali de l'aménagement du Litani au Liban (1965). Travaux, mai 1965.

LACrolx (R.). - Études préliminaires sur les ouvrages de prise d'eau pour centrale nucléaire en bordure de la Méditerranée La Houille Blanche, $\mathrm{n}^{\circ} 7 / 8$ - 1975.

MANIER et LEPETIT, - Ouvrages de prise et rejet d'eau pour la centrale nucléaire de Gravelines. La Houille Blanche, n $7 / 8$ 1975

MAssov (M.). - Pétrophysique de la craie (1973). Bull. Liaison Labo P.ect-Ch.. n" spécial V.

Plouviez. Fauchart et Hueber. - Essais au puits de Sangatte: premières applications au calcul du revêtement du tunnel sous la Manche (1974). EDIRAP. Paris.

RAT (M.). - Écoulement et répartition des pressions interstitielles autour des tunnels (1973). Bull. Liaison Labo P.-et-Ch. n" 68.

\title{
Discussion
}

\author{
Président: M. J.-C. LEBRETON
}

M. le Président remercie les deux conférenciers de leur exposé fort documenté et poursuit: M. BELLIN nous a fait un peu un cours d'architecture sur les circuits hydrauliques des centrales littorales et M. DufFaut a esquissé une comparaison entre les grands tunnels des usines hydroélectriques et ceux qui vont être sans doute réalisés pour les circuits d'eau de certaines centrales implantées sur nos côtes.

On dit souvent - ce qui permet de cadrer les choses - qu'une prise d'eau pour quatre tranches de chacune 1 million de $\mathrm{kW}$ absorbe un débit de l'ordre du débit moyen de la Seine à Paris. Il est important d'avoir ce chiffre en tête pour se représenter les ouvrages de canalisation nécessaires pour transiter de tels débits. Et pourtant, dans le contexte maritime, ce chiffre est modeste puisque, en Manche par exemple, les débits de marée atteignent, sur une frange littorale de quelques kilomètres, plusieurs dizaines et parfois plusieurs centaines de milliers de $\mathrm{m}^{3} / \mathrm{s}$. Pour caractériser l'importance de telles prises d'eau, j'aimerai qu'on indique, outre leur débit, l'importance des travaux de génie civil (déroctages, notamment) qu'elles exigent. Il est vrai que, pour une centrale implantée sur le littoral - et vous l'avez d'ailleurs fort bien dit il est difficile de séparer les travaux de déroctage et remblaiement imputables au circuit d'eau proprement dit, de ceux imputables à la plateforme elle-même; néanmoins, il serait intéressant de séparer les frais d'établissement et les volumes de déblais afférents d'une part, aux travaux liés au calage de la plateforme et, d'autre part, aux ouvrages d'amenée et de rejet d'eau proprement dits. On ne parlera peut-être pas beaucoup des aspects économiques aujourd'hui; j'indiquerai simplement que ces prises d'eau arrivent a des coûts de l'ordre de 200 à 400 millions de franes lourds, soit au moins $10 \%$ - et mème plus - du coût d'une tranche de $1000 \mathrm{MW}$. Les frais d'établissement élevés des centrales conduisent à être très exigeants quant à leur fiabilité; en gros, l'immobilisation durant un mois d'une centrale peut conduire à un manque à gagner de l'ordre de 40 millions de francs : ainsi quatre ou cing mois d'immobilisation représentent l'équivalent du coût de la prise d'eau.

Mais, sans doute, avez-vous de nombreuses questions à poser aux conférenciers; j'ouvre donc la discussion.

Dans son exposé, observe M. LAJEAT (E.D.F., Equipement), M. BELLIN a présenté les problèmes de «taches chaudes» du rejet et la position relative des prises d'eau sur des vues en plan. Ne serait-il pas intéressant de parler également de ce qui se passe dans des plans verticaux?

En ce qui concerne la répartition verticale des températures, répond $M$. BELLIN, il convient de distinguer ce qui se passe dans une mer à marée - comme la Manche et l'Atlantique - et dans une mer sans marée comme la Méditerranée. Dans le cas de la Manche, la profondeur est relativement faible et, du seul fait des courants de marée, il y a un brassage suffisant pour que les températures soient sensiblement les mêmes sur toute une verticale; du fait de cette absence de stratification, il n'y a aucun intérêt à réaliser, par exemple, des prises d'eau en profondeur et des rejets en surface. Par contre, en Méditerranée, mer profonde où il n'y a pas de marée, il y a un phénomène de stratification très net. En été, la température des eaux en surface est d'une vingtaine de degrés, alors qu'au-dessous de la cote -40 , I'eau se trouve à 12 . 13 ou $14^{\circ}$. De ce fait, en Méditerranée, les eaux chaudes rejetées au niveau de la centrale ont tendance à flotter et à rester en surface; au départ, l'aspect général de la tache d'eau chaude est commandé par le phénomène de jet flottant; lorsqu'on s'éloigne suffisamment de l'ouvrage de rejet, la tache d'eau chaude se développe en fonction des vents et en fonction des courants locaux. On pourrait être tenté, en Méditerranée, d'établir des ouvrages de prise d'eau en profondeur et des rejets en surface. Nous n'avons pas encore terminé l'étude de cette disposition. D'autre part, tous les sites pour lesquels la cote $-30,-50$ se trouve proche de la côte, sont en général des sites touristiques et cela pose d'autres problèmes..

Lorsque la plateforme de la centrale exige une grande excavation sur un littoral escarpé, comment prenez-vous en compte le risque d'éboulement de la falaise artificielle dominant l'usine? demande M. A. LEBRETON (Direction de l'Electricité).

Les pentes et les risbermes des talus sont choisis, répond $M$ BELLIN, de manière que les éboulements risquant de se produire n'entraînent pas la chute de matériaux sur la centrale; d'ailleurs un tel incident ne poserait pas, semble-t-il, de problèmes majeurs de sécurité car les structures essentielles de la centrale sont calculées pour résister à des impacts plus importants, tels que celui d'un avion par exemple. 
En effet, précise $M$. Duffaut, un éboulement superficiel ne provoquerait guère de dégâts. Le problème grave est celui de la protection de la centrale contre un glissement d'ensemble des terrains - rocheux ou non - comme il s'en produit parfois en montagne. Il doit être étudié dans chaque cas.

C'est là une question relativement classique pour les ingénieurs des exploitations minières à ciel ouvert (telle que la « découverte " de Decazeville). Il faut choisir la pente des talus en tenant compte de leur hauteur et de la cohésion du sol ou du rocher. En période humide, un système de drainage bien étudié est la meilleure protection contre les glissements de terrain sous l'action des "pressions de courant".

M. BIAlek (Ponts et Chaussées, Dieppe) revient sur la question de la diffusion des eaux chaudes rejetées. Sur quelles bases peuton affirmer que le mélange se fait intimement en Manche et s'avère généralement inexistant en Méditerranée?

La répartition verticale très différente des températures en Manche et en Méditerranée, répond M. Boulot (L.N.H., Chatou), résulte essentiellement

I $^{\circ}$ des vitesses des courants de marée, fortes dans le premier cas (de l'ordre d'un noud) et faibles dans le second;

$2^{\circ}$ des profondeurs beaucoup plus faibles en Manche.

Cela entraîne une bonne homogénéisation des températures en Manche et une stratification en Méditerranée (où une thermocline apparaît toujours en été)

D'autre part, les études montrent qu'il n'y aura pas d'hétérogénéité spatiale notable des températures, sauf au voisinage immédiat des ouvrages de rejet.

M. R. LOIRE (TRAMCO S.A.) pose la question suivante :

M. BELLiN n'a pas parlé des centrales au large (off shore). On semble souvent éliminer celles-ci a priori en invoquant un coût trop élevé. Mais ne permettent-elles pas de faire l'économie de travaux importants (les $7000000 \mathrm{~m}^{3}$ de déroctage de PALuel, par exemple) ou d'éliminer des sujétions de fonctionnement et d'entretien des prises d'eau et canaux d'amenée? l'étude comparative vaut la peine d'être faite.

Comment est résolu le problème de la source froide dans ces centrales off shore?

On peut classer les centrales off shore, répond M. BELLIN, en deux grandes catégories

a) les centrales implantées sur une île artificielle, établies à 5 ou $10 \mathrm{~km}$ des côtes:

b) les centrales flottantes ou échouées qui sont construites à terre par exemple dans un chantier naval, remorquées et enfin installées sur un site donné.

En ce qui concerne le premier type de centrale, la réalisation d'une île est un ouvrage de génie civil important qui nécessite des études et un délai d'exécution assez longs; outre son prix, cette île a l'inconvénient de devoir être mise à la disposition des constructeurs de la centrale dès le début du chantier; elle ne commencera donc à s'amortir que sept ou huit ans après, ce qui entraîne des intérêts intercalaires élevés. Ces centrales sont très onéreuses. Une fois installées, les centrales construites sur une île artificielle - et même la centrale flottante - vont être plus ou moins coupées de la terre, d'où des suppléments de coûts et des aléas importants pour l'évacuation de l'énergie et pour l'accès du personnel et du matériel d'exploitation.

En ce qui concerne les centrales qui sont fabriquées dans un chantier et remorquées, je donnerai quelques chiffres. La filiale de Westinghouse qui construit cette centrale sur le chantier de Jackson fait savoir, au départ, qu'elle n'engagerait une telle opération que dans la mesure où elle aurait six commandes; elle estime que, pour être rentable, le chantier devrait construire quatre à six centrales par an. Sur le marché américain, ce type de centrale peut s'avérer intéressant car les Américains commandent, en gros, une quarantaine de " tranches " par an. Il n'en est pas de même sur le marché français, où nous ne commanderons peut être que six tranches par an (et toutes nos centrales ne devront pas être des centrales flottantes). Peut-être, à l'échelle européenne, la solution serait-elle plus intéressante si une entente intervenait entre les divers pays intéressés.

Concernant votre dernière question, il semble que la centrale flottante bénéficierait plus facilement de fonds à la cote -12 , - 15; donc, ses ouvrages d'adduction d'eau pourraient être simplifiés. Par contre, la dispersion des calories et la qualité de la source froide ne seraient guère améliorées par rapport à une centrale implantée sur le littoral au droit de la centrale flottante.
En Méditerranée, où il n'y a pas de courants de marée, quelle est l'importance du phénomène d'up-welling qui peut se produire sous l'action de la tramontane? demande M. EzRATY?

En ce qui concerne Port-la-Nouvelle, répond M. BELLIN, une étude, aussi précise que possible, de tous les phénomènes à caractère aléatoire influant sur la source froide est en cours. Malheureusement, ces phénomènes, dans le passé, n'ont guère été observés et sont plus ou moins connus; on aura certainement de bien meilleures connaissances sur ce point au moment où démarrera cette installation aujourd'hui en projet.

Toujours en ce qui concerne Port-la-Nouvelle, demande M. EzRATY, quel est l'ordre de grandeur du gradient longitudinal de température à la sortie de l'usine, c'est-à-dire sur le canal de rejet?

Entre la sortie de la centrale et l'ouvrage de rejet proprement dit, précise M. BELLIN, les échanges atmosphériques sur le canal sont très faibles et les températures pratiquement constantes. La différence de température entre le point de prise d'eau et le point de rejet de la centrale sera de l'ordre d'une dizaine ou d'une douzaine de degrés. Immédiatement à la sortie de l'ouvrage de rejet, se place le phénomène de jet que nous essayons d'optimiser et qui permettra d'entraîner les eaux froides environnantes au sein des eaux chaudes que nous rejetons et d'arriver à un certain mélange; on passe progressivement, mais assez rapidement, de l'isotherme $+12^{\circ}$ à l'isotherme $+3{ }^{\circ} \mathrm{C}$ et ensuite de $+3{ }^{\circ} \mathrm{C}$ à $+1^{\circ} \mathrm{C}$ : la tache thermique est alors très grande.

A la demande de $M$. le Président, $M$. Boulor revient sur le phénomène d'up-welling. Ce phénomène n'est pas gênant pour la centrale; il va amener de l'eau froide en surface, donc diluer l'eau échauffée dans de l'eau plus froide, ce qui est relativement favorable. Malheureusement, il se produit très rarement - quelques fois pendant l'été - et on ne pourra en profiter qu'occasionnellement pour refroidir le rejet qui, comme on l'a dit, se présente ici sous la forme d'un jet flottant.

M. MilLot (E.D.F.-D.A.F.E.C.O.) pose la question ci-après :

Les profondeurs de prise mentionnées sont de 12 à $15 \mathrm{~m}$; quels sont les critères qui conduisent à cette profondeur. Des prises d'eau en surface ou à plus faible profondeur ont-elles été envisagées?

Si l'on appelle $t$ la profondeur - distance du fond au niveau moyen de la surface - on estime, précise M. BELlin, que pour éviter toute interaction avec la zone de déferlement d'une houle de hauteur $H$, la prise doit être implantée dans une zone où $t>2 \mathrm{H}$; or, dans la plupart des sites que nous avons étudiés, les houles atteignent une amplitude de 5 à $6 \mathrm{~m}$. D'autre part, pour ne pas risquer d'entraîner le sable ou les autres matériaux du fond, son seuil doit être à une distance de l'ordre de $0,3 t$ du fond marin ( $t$ étant la profondeur moyenne). Finalement, pour les sites considérés, on arrive à des profondeurs $t$ d'une douzaine de mètres, le seuil de la prise étant placé à $3 \mathrm{~m}$ au-dessus du fond.

Ce problème, observe $\mathrm{Ni}$. le Président, est aussi lié aux vitesses maximales qu'on peut admettre dans la prise d'eau, en raison de questions biologiques et de la nécessité notamment de ne pas aspirer des poissons; c'est en fonction d'une vitesse-seuil que l'on se donne que, finalement, se trouve dimensionnée la surface d'entrée; si cette vitesse est inférieure à $0,50 \mathrm{~m}$, elle implique une grande surface d'entrée qui nécessite une épaisseur elle-même assez grande (elle peut atteindre 7 à $8 \mathrm{~m}$ ).

M. le Président AUBERT, Ingénieur Général des Ponts et Chaussées (e.r.) intervient en ces termes :

En ce qui concerne les prises d'eau de mer - par exemple dans le cas des plages de sable - je pense que l'E.D.F. a intérêt à éviter de donner prise à des critiques concernant les ouvrages correspondants; le principe même des usines nucléaires étant déjà suffisamment contesté par le public.

A cet égard, le système de l'ouvrage ensouillé me paraît devoir être préféré à celui du canal à ciel ouvert délimité par deux digues en enrochements. Dans ce dernier cas, deux objections sont en effet à redouter. La première concerne l'arrêt des transports littoraux, la deuxième étant d'ordre esthétique.

Je pense que le moment n'est pas venu d'évoquer les conditions de construction d'un tel ouvrage ensouillé dans une plage battue par des lames déversantes.

Vous avez parfaitement raison, observe M. le Président, de signaler l'aspect délicat et peut être psychologique que peut soulever l'emploi, ici, des canaux à écoulement libre. Je dirais à l'avantage de ces solutions que, sous réserve de prendre des précautions 
quant à la stabilité du littoral, ces ouvrages ne sont pas mal reçus par l'opinion car ils s'apparentent assez aux ouvrages des ports maritimes (j'irais même jusqu'à dire qu'on nous les a suggérés et nous nous promettons de regarder, cas par cas, la compatibilité d'un ouvrage portuaire ou d'un port de plaisance avec de tels ouvrages de prise d'eau); pourquoi ne pas essayer de joindre l'utile à l'agréable. Quant au pont-canal, là encore je pense que les représentants de M. LACROIX le diront, il n'est pas impensable, et on nous a même suggéré de jumeler avec ce pont-canal une plateforme portuaire à l'endroit de la prise d'eau ce qui constituerait un véritable petit port en mer.

Je crois donc que ces ouvrages peuvent ne pas avoir l'impact psychologique défavorable que vous redoutiez.

Je voudrais ajouter, dit M. ALEMANy (E.D.F., Direction de l'Equipement), qu'outre les avantages que vient d'indiquer $M$. LEBRETON au sujet des ouvrages d'eau (canal d'amenée et de rejet) à ciel ouvert : port de plaisance, etc., la possibilité de recevoir directement sur le site, par bateau, les colis lourds et encombrants que constituent les matériels nucléaires: cuve et générateurs de vapeur non transportables par fer et difficilement par route, sinon au prix d'aménagements très coûteux. L'aménagement d'un appontement valoriserait largement le coût de ces ouvrages. Cette conception est également valable dans le cas d'une prise d'eau au large raccordée par un pont-canal du type à « voussoirs » supportant les conduites d'eau reliées aux condenseurs.

A la demande de M. le Président, M. Duffaut, résume ainsi son opinion sur la possibilité de réaliser des galeries sous-marines en terrains meubles:

En principe, il n'y a pas de problème à condition de congeler le terrain à l'avance; mais cela coûte très cher... La difficulté est plus grande que pour la construction du Métro à Paris par exemple, car il convient d'éviter à tout prix l'envahissement du chantier par les eaux marines; le respect de la condition de sécurité au « renard » devient, ici, une religion.

Peut-être y a-t-il un espoir du côté des machines foreuses, entièrement automatiques, qui pourront fonctionner sous l'eau sous charge et sans intervention humaine, ainsi que le prévoit un procédé japonais: il y a encore un homme au fond, mais qui n'a qu'une fonction de surveillance et que l'on pourrait probablement supprimer.

On pourrait songer - ainsi que le suggère M. EzRATY - à utiliser le lancement et l'échouage sur le fond des éléments du canal ainsi que cela se pratique pour les tunnels routiers sous- fluviaux mais, apparemment, il est très difficile d'utiliser ce procédé dans la zone de déferlement de la houle.

Au sujet des positions respectives de l'ouvrage de prise et de l'ouvrage de rejet dans une mer qui, comme la Manche, est bien homogène, $M$. CHABERT D'HIËRES demande : quelle est la raison qui fait placer la prise d'eau au large et le rejet à la côte?

M. NURY (E.D.F., Equipement) répond en ces termes :

La disposition actuellement retenue pour les ouvrages d'eau avec prise au large et rejet à la côte permet de :

- résoudre le problème de l'amortissement de la houle;

- d'éviter l'entraînement des matériaux en suspension;

elle présente par contre l'inconvénient:

- de nécessiter l'implantation de la prise à des profondeurs importantes (vers - $10 \mathrm{~m}$ « cote marine »);

- de laisser la prise sans surveillance et done de présenter un risque d'entraînement de corps flottants (bancs de poissons, notamment).

L'inversion de ces dispositions (prise à la côte par chenal et rejet au large par galerie) permet de supprimer ces inconvénients; le rejet peut se faire à des cotes plus faibles ( $-5 \mathrm{~m}$ cote marine) et, dans certaines conditions, la dilution peut également être meilleure.

En contrepartie, on risque d'avoir des difficultés pour :

- amortir la houle (les filtres rotatifs ne tolèrent pas des oscillations de plan d'eau de plus de $20 \mathrm{~cm}$ );

- éviter l'entraînement de sables.

On peut aussi, observe M. le Président, porter au passif de la solution "prise à la côte et rejet au large ", l'augmentation de la durée de séjour des micro-organismes marins dans l'eau à la température du condenseur.

M. Canavelis (Bergeron S.A.) ajoute que le choix entre les deux dispositions susvisées peut avoir une influence sur le régime transitoire des pompes de circulation des condenseurs et il peut en résulter diverses complications de Génie Civil à étudier dans chaque cas. En outre, dans les conduites parcourues par de l'eau froide, les risques d'incrustations, de dépôts de moules ou d'algues sont plus grands que dans les canalisations d'eau chaude en aval du condenseur.

En raison des exigences de l'horaire, M. le Président clôt la discussion en remerciant tous ceux qui ont contribué à l'animer et tout spécialement MM. BELlin et DUFFaUT.

Il donne ensuite la parole successivement à M. MAINIER et à M. LEPETIT pour l'exposé de leur Communication. 